\title{
Prevalência de desnutrição em idosos institucionalizados: uma revisão crítica sistemática
}

\section{Prevalence of malnutrition in elderly institucionalized: a critical review systematized}

\author{
Larissa Praça Oliveira ${ }^{1}$, Natália Louise Araújo Cabral ${ }^{2}$, Diôgo Vale ${ }^{3}$, Clélia Oliveira Lyra ${ }^{4}$, Kenio Costa Lima ${ }^{4}$
}

1. Doutoranda em Saúde Coletiva pela Universidade Federal do Rio Grande do Norte, Brasil, 2. Mestranda em Saúde Coletiva pela Universidade Federal do Rio Grande do Norte, Brasil, 3. Departamento de Nutrição do Centro de Ciências da Saúde da Universidade Federal do Rio Grande do Norte, Brasil, 4. Programa de Pós-graduação em Saúde Coletiva do Departamento de Nutrição da Universidade Federal do Rio Grande do Norte, Brasil.

\section{Resumo}

Introdução: A desnutrição é um importante distúrbio nutricional em idosos institucionalizados por estar associada ao aumento da mortalidade e da susceptibilidade às infecções e à redução da qualidade de vida. A avaliação periódica do estado nutricional é fundamental para a promoção ou reabilitação da saúde em idosos. Para tanto, os métodos mais utilizados são os antropométricos, bioquímicos, de consumo alimentar e a Mini Avaliação Nutricional. Este trabalho visa identificar a prevalência de desnutrição em idosos institucionalizados e os métodos diagnósticos utilizados para determinar essa deficiência nutricional. Metodologia: A revisão foi realizada a partir das bases eletrônicas LILACS, IBECS, SCIELO, Medline, PubMed e Scopus. A seleção dos estudos foi feita considerando artigos originais completos disponíveis on-line, em inglês, espanhol e português, publicados entre 1992 e 2013. Resultados: Todos os estudos foram desenvolvidos em mais de uma instituição asilar. Destes, 71,4\% (n=15) consideraram como idosos pessoas com idade mínima de 65 anos As prevalências de desnutrição variaram de 6,3\% a 81,7\% dependendo do método, indicadores e critérios utilizados para o diagnóstico. Todos os estudos utilizaram o Índice de Massa Corporal para diagnóstico do estado nutricional, quer como método isolado, ou associado a outro método, utilizando distintos valores de referência. Conclusões: Existe uma grande variabilidade na prevalência de desnutrição em idosos institucionalizados, sendo a Mini Avaliação Nutricional o método mais utilizado para o diagnóstico. Mais estudos são necessários para subsidiar o desenvolvimento de instrumentos que permitam uma avaliação nutricional mais robusta e que possa ser utilizado tanto para acompanhamento clínico quanto para a investigação epidemiológica.

Palavras chave: Idosos. Desnutrição. Asilo para idosos. Revisão Sistemática.

\begin{abstract}
Introduction: Malnutrition is an important nutritional disorder among institutionalized elderly due to its association with increased mortality and susceptibility to infections and reduced quality of life. Periodic assessment of nutritional status is essential for the promotion of health and rehabilitation in the elderly. For that purpose, the most widely used methods are the anthropometric, biochemical, dietary intake and Mini Nutritional Assessment. This article aims at identifying the prevalence of malnutrition in institutionalized elderly and the diagnostic methods used to determine this nutritional deficiency. Methods: A retrospective review was conducted from the electronic databases Lilacs, IBECS, SciELO, MEDLINE, PubMed, and SCOPUS. Studies were selected considering their full availability on-line in English, Spanish and Portuguese and original articles published between 1992 and 2013 . Results: All studies were conducted in more than one nursing home. Among these, $71.4 \%(n=15)$ elderly subjects were considered aged after being at least 65 years. The prevalence of malnutrition ranged from 6.3\% to $81.7 \%$ depending on the method, indicators and criteria used for diagnosis. All studies used the Body Mass Index for diagnosing nutritional status, either as an isolated method, or combined with another method using different benchmarks. Conclusion: There is a high prevalence of malnutrition in institutionalized elderly, and the Mini Nutritional Assessment method was most widely used for diagnosis. More studies are needed to support the development of tools to allow a more robust nutritional assessment so that it may be used both for clinical monitoring and for epidemiological research.
\end{abstract}

Keywords: Aged. Malnutrition. Institutionalized elderly. Review

\section{INTRODUÇÃO}

Nos países desenvolvidos e em desenvolvimento, a rede de cuidados ao idoso, especialmente ao idoso frágil, inclui o domicílio e as modalidades de assistência e de cuidado, como uma das principais formas de atenção à saúde desta população ${ }^{1}$. Entre estas modalidades, as instituições asilares, sejam elas de caráter público, filantrópico ou particular, vêm ganhando destaque em virtude do aumento repentino do número dessas instituições que, apesar de desempenharem importante função na sociedade, podem trazer sérias repercussões biopsicossociais às pessoas idosas que nelas residem².

No Brasil, as instituições asilares são estabelecimentos que visam 
acolher pessoas que possuem 60 anos ou mais, dependentes ou não, que indispõem de condições para permanecer com a família e/ou em seu domicílio, devendo atender às necessidades de cuidados com a saúde, alimentação, higiene, repouso e lazer de seus residentes ${ }^{3}$, e, se configuram em locais indispensáveis para o cuidado e assistência à saúde da pessoa idosa.

No entanto, o processo de institucionalização também pode provocar modificações na rotina dos idosos, especialmente no que concerne ao comportamento alimentar, podendo levar a alterações dos hábitos alimentares e potencializar a fragilidade da saúde desses indivíduos ${ }^{4}$. Além disso, as alterações fisiológicas inerentes ao envelhecimento, às morbidades e à polifarmácia, contribuem para o comprometimento do consumo de alimentos e a absorção dos nutrientes, aumentando o risco de desenvolvimento de deficiências nutricionais nesses idosos. Dessa forma, a desnutrição se apresenta como um dos problemas mais preocupantes na população idosa institucionalizada ${ }^{5}$.

A Desnutrição Proteico-Calórica (DPC) é o distúrbio nutricional mais importante observado nesta população e está associada ao aumento da mortalidade e da susceptibilidade às infecções e à redução da qualidade de vida. Entretanto, muitas vezes, a DPC é vista, erroneamente, como parte do processo normal de envelhecimento, sendo com frequência ignorada pelas equipes de saúde das instituições ${ }^{6}$.

A presença da desnutrição pode interferir de maneira significativa na evolução de enfermidades crônicas e agudas dos idosos. A DPC está associada ao agravo de doenças crônicas, dificuldade de cicatrização de úlceras de pressão ou feridas e aumento dos períodos de hospitalização, gerando custos para o Estado e diminuindo a qualidade de vida dos idosos ${ }^{7}$.

Por isso, a avaliação periódica do Estado Nutricional (EN) para a identificação do risco de desnutrição, faz-se necessária nos idosos institucionalizados, sendo os métodos antropométricos, bioquímicos, de consumo alimentar e a Mini Avaliação Nutricional (MAN) os mais comuns $8,8,10,11$.

Diante dos aspectos abordados, a desnutrição deve ser considerada um relevante problema de saúde pública em idosos e pode estar relacionada com a diminuição da expectativa de vida e ao prognóstico desfavorável da evolução de quaisquer morbidades $^{6}$.

Assim, o objetivo desta revisão crítica sistemática consiste em identificar a prevalência de desnutrição em idosos institucionalizados e os métodos diagnósticos utilizados para determinar essa deficiência nutricional.

\section{METODOLOGIA}

A revisão crítica e sistemática dos estudos envolvendo a temática da desnutrição em idosos institucionalizados foi realizada no período compreendido entre Março e Abril de 2013, utilizando bases de dados eletrônicas nacionais e internacionais, sendo estas: Literatura Latino-Americana e do Caribe em Ciências da Saúde (LILACS); Índice Bibliográfico Espanhol de Ciências da Saúde (IBECS), Scientific Electronic Library Online (Scielo); PubMed e Scopus.

Dessas bases, foram selecionados apenas os estudos originais completos disponíveis on-line, publicados em inglês, espanhol e português, os quais estimavam a prevalência de desnutrição em idosos institucionalizados. Para a pesquisa, foram incluídos artigos publicados entre os anos de 1992 e 2013, sendo considerados, nas bases nacionais, os seguintes descritores: Idosos; Saúde de idosos institucionalizados; Asilos para idosos; e Instituição de Longa Permanência para Idosos. Esses descritores foram combinados com palavras chaves relacionados ao estado nutricional como: estado nutricional, desnutrição, e nutrição. Método semelhante foi utilizado nas bases internacionais, por meio dos descritores: Elderly, Institutionalized elderly e Nursing home, sendo estes combinados com as seguintes palavras chave: malnutrition, nutrition, undernutrition e nutritional status.

A Biblioteca Virtual em Saúde (BVS) foi utilizada para as buscas dos artigos publicados no Lilacs, IBECS e Scielo. Os descritores supracitados foram combinados com operadores booleanos AND, OR, NO, para verificar se existia diferença no número de artigos encontrados.

Inicialmente, foi realizada a leitura dos títulos e resumos (abstracts) para identificação daqueles que se enquadrariam dentro dos critérios estabelecidos para esta revisão. Os critérios de inclusão compreenderam: a) idosos residentes em instituições asilares, com idade mínima de 60 anos; b) possuir amostra probabilística; c) apresentar dados originais de prevalência de desnutrição; e d) apresentar concordância dos estudos transversais com os critérios do Strengthtening the Reporting of Observational Studies in Epidemiology (STROBE ${ }^{12}$ ).

O STROBE consiste em uma lista de verificação contendo 22 itens relacionados a informações que deveriam estar presentes no título, resumo, introdução, metodologia, resultados e discussão de pesquisas e publicações na área de epidemiologia que envolve estudos com delineamento observacional, visando uma descrição mais precisa e completa do estudo apresentado ${ }^{12}$. Foram excluídos do estudo os artigos de revisão.

Artigos de estudos multicêntricos com populações de continentes diferentes e que apresentassem o resultado caracterizando a população de cada um dos países, em seus respectivos continente, foram apresentados de forma separada nos resultados, para que a comparação das prevalências entre os continentes fosse possível.

Todas as pesquisas foram realizadas por dois pesquisadores, de forma independente e com cegamento, levando em consideração os descritores definidos para esta revisão, bem como os critérios de inclusão e exclusão. Após isso, os pesquisadores, em consenso, definiram quais artigos iriam compor esta revisão. Caso houvesse discordância na seleção dos artigos, um terceiro avaliador seria consultado ${ }^{13}$. 
Os artigos selecionados foram caracterizados quanto: a) país em que foi desenvolvido; b) ano de publicação; c) periódico de publicação; d) os indicadores de estado nutricional adotados; e) critério para diagnosticar desnutrição; f) prevalência de desnutrição estimada. Para caracterização e ordenação dos dados utilizou-se a planilha eletrônica do Microsoft Excel ${ }^{\circledR}$.

\section{RESULTADOS}

Um total de 1.362 artigos foram encontrados nas bases de dados escolhidas para o estudo, seguindo as estratégias de busca previamente definidas entre os pesquisadores. Destes, 538 artigos foram excluídos, a partir da leitura do título e do resumo, por não apresentarem a temática do estudo e não abordarem exclusivamente a população idosa e institucionalizada. Assim, um total de 824 artigos foram apreciados pelos pesquisadores que, em consenso, excluíram aqueles em duplicata $(n=309)$, os que não atenderam aos critérios do $\operatorname{STROBE}^{12}(n=56)$ e os que não dispunham do texto completo $(n=438)$. Portanto, apenas 21 artigos foram selecionados para discussão nesta revisão. $A$ Figura 1, a seguir, apresenta o diagrama do fluxo da seleção final dos artigos encontrados nas bases pesquisadas.

Os estudos revelaram predominância de sujeitos do sexo feminino na composição da amostra. Apenas um estudo ${ }^{14}$
$(4,8 \%)$, apresentou a amostra com uma maior ocorrência de idosos do sexo masculino. Três estudos ${ }^{11,15,16}$ (14,3\%) não classificaram a amostra quanto ao sexo. A maioria das pesquisas, $71,4 \%$ ( $n=15)^{8,10,11,14,16,17,18,19,20,21,22,23,24,25,26}$ considerou como idosos os sujeitos com idade mínima de 65 anos, e apenas seis $^{15,27,28,29,30,31}(28,6 \%)$ a idade mínima considerada foi 60 anos. Esse dado mostra que a maior parte da população estudada residia em países desenvolvidos, onde são denominados idosos aqueles com idade superior a 65 anos.

Todos os estudos foram desenvolvidos em mais de uma instituição asilar, sendo 14 desses realizados no continente Europeu $(66,7 \%)$, quatro no continente Americano (19,0\%), um no continente Asiático (4,8\%), um na África (4,8\%) e um na Oceania $(4,8 \%)$, como está apresentado na tabela 1.

Houve grande variação na prevalência de desnutrição entre os estudos, devido aos métodos, aos indicadores e aos critérios utilizados para diagnosticar a desnutrição. A menor prevalência $(6,3 \%)$ foi obtida em um estudo italiano ${ }^{11}$ que utilizou o índice de Massa Corporal em associação ao MAN para o diagnóstico da desnutrição.

O estudo de Menezes e Marucci ${ }^{27}$, realizado no Brasil, foi o que encontrou a maior prevalência de desnutrição $(81,7 \%)$,

Figura 1. Diagrama do fluxo da pesquisa bibliográfica e seleção dos artigos para a revisão sistemática

1. Estratégia de busca

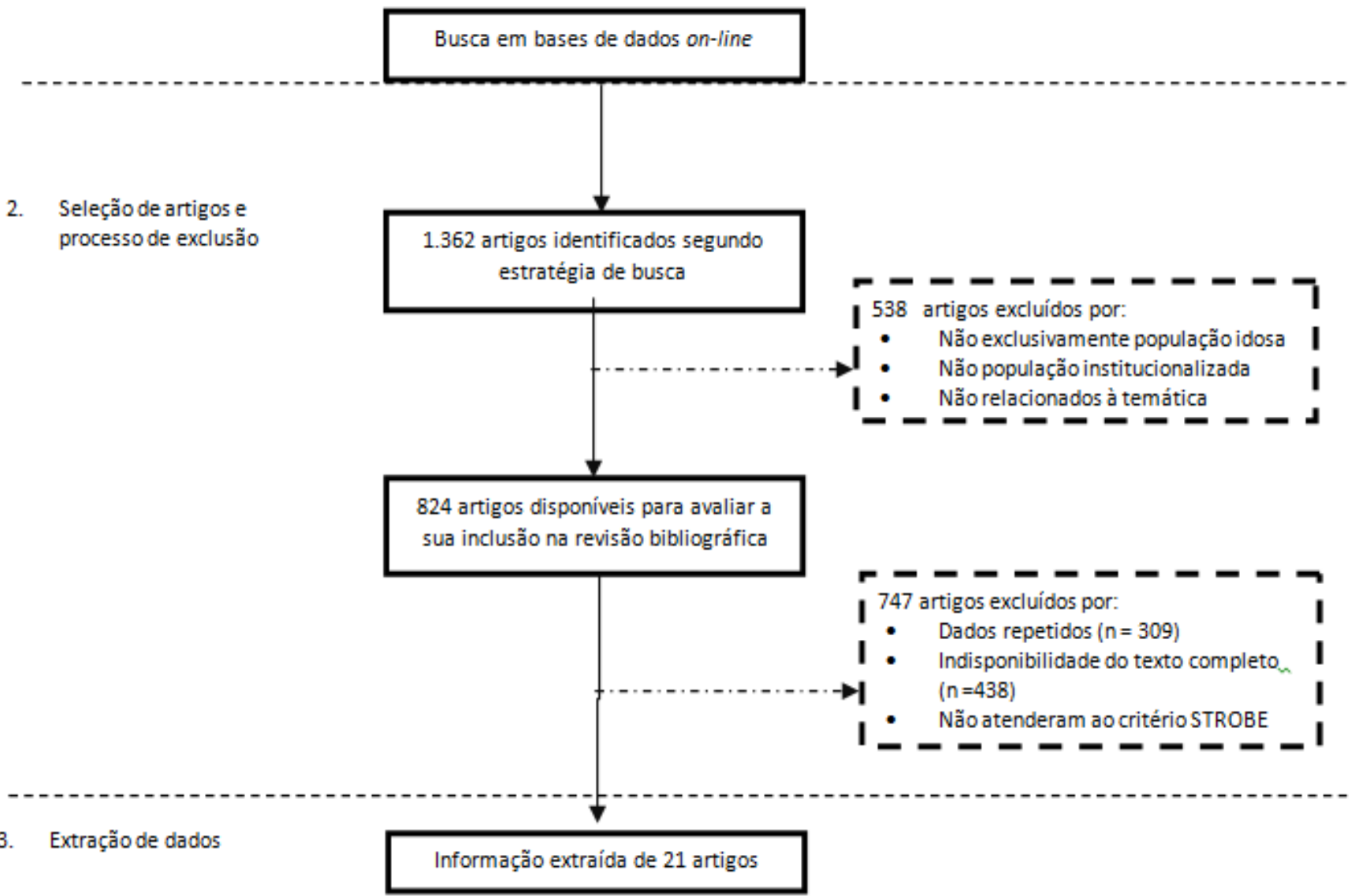


138 Prevalência de desnutrição em idosos institucionalizados: uma revisão crítica sistemática

Tabela 1. Características das metodologias empregadas nos estudos de prevalência de desnutrição em idosos institucionalizados.

\begin{tabular}{|c|c|c|c|c|c|c|}
\hline $\begin{array}{l}\text { Autores do Estudo e Ano de } \\
\text { publicação }\end{array}$ & Periódico & Continente/ País & Método & $\begin{array}{l}\text { Indicadores do Esta- } \\
\text { do nutricional }\end{array}$ & $\begin{array}{l}\text { Critério para desnu- } \\
\text { trição }\end{array}$ & Prevalência \\
\hline Hewitt, et al, 2006 & West Indian Med J & Americano/Guiana & Antropométricos & $\begin{array}{l}\mathrm{IMC}^{\mathrm{b}} \\
\mathrm{PB}^{\mathrm{c}} \\
\mathrm{PP}^{\mathrm{d}}\end{array}$ & $\begin{array}{c}<18,5 \mathrm{~kg} / \mathrm{m} 2 \\
<24 \mathrm{~cm} \text { (pessoas de } \\
\text { ascendência Africana), } \\
<23 \mathrm{~cm} \text { (para homens } \\
\text { de ascendência } \\
\text { indiana), }<22 \mathrm{~cm} \\
\text { para (mulheres de } \\
\text { ascendência Indiana) } \\
<30,5 \mathrm{~cm}\end{array}$ & $\begin{array}{l}29,3 \% \text { públicas } \\
19,2 \% \text { privadas }\end{array}$ \\
\hline Gaskill et al, 2008 & $\begin{array}{c}\text { Australasian Journal on } \\
\text { Ageing }\end{array}$ & Australiano/Austrália & Triagem Nutricional & ASG $\mathrm{g}$ & - & $49,5 \%$ \\
\hline Lelovics et al, 2009 & Nutr Hosp & Europeu/Espanha & $\begin{array}{l}\text { Antropométricos } \\
\text { Triagem Nutricional }\end{array}$ & $\begin{array}{c}\mathrm{IMC}^{\mathrm{b}} \\
\text { Perda de peso invo- } \\
\text { luntária } \\
\mathrm{MUST}^{\mathrm{h}}\end{array}$ & $\begin{array}{c}<20,0 \mathrm{~kg} / \mathrm{m}^{2} 5-10 \% \\
\text { peso em } 3-6 \text { meses }>2 \\
\text { pontos }\end{array}$ & $\begin{array}{c}19,2 \% \\
6,7 \% \\
38,2 \%\end{array}$ \\
\hline Carlsson et al, 2009 & $\begin{array}{c}\text { Archives of Gerontology and } \\
\text { Geriatrics }\end{array}$ & Europe/Suécia & Triagem Nutricional & $\mathrm{MAN}^{\mathrm{a}}$ & $<17$ & $17,4 \%$ \\
\hline $\begin{array}{l}\text { Bourdel-Marchasson et al, } \\
2009\end{array}$ & Nutrition & Europeu/França & Triagem Nutricional & $\mathrm{MAN}^{\mathrm{a}}$ & $<17$ & $19,1 \%$ \\
\hline Smoliner et al, 2009 & British Journal of Nutrition & Australiano/Austria & Triagem Nutricional & MAN $^{a}$ & $<17$ & $22,8 \%$ \\
\hline Langiano et al, 2009 & Igiene e sanità pubblica & Europeu/Itália & $\begin{array}{l}\text { Triagem Nutricional } \\
\text { Antropométricos }\end{array}$ & $\begin{array}{l}\text { MAN }^{a} \\
\text { IMC }^{b}\end{array}$ & $\begin{array}{c}<176,3 \% \\
<20,0 \mathrm{Kg} / \mathrm{m}^{2}\end{array}$ & $\begin{array}{c}13,0 \% \\
6,3 \%\end{array}$ \\
\hline Menezes e Marucci, 2010 & Rev. Bras. Geriatr. Gerontol. & Americano/Brasil & Antropométricos & $\begin{array}{l}\mathrm{IMCB}^{\mathrm{b}} \\
\mathrm{PCT}^{\mathrm{f}} \\
\mathrm{PMB}^{\mathrm{e}}\end{array}$ & $\begin{array}{l}\leq \mathrm{P} 25(\dot{C}) \\
\leq \mathrm{P} 25 \\
\leq \mathrm{P} 25\end{array}$ & $\begin{array}{l}53,1 \% \\
26,9 \% \\
81,7 \%\end{array}$ \\
\hline Laura et al, 2010 & $\begin{array}{c}\text { Nutricion Clinica y Dietetica } \\
\text { Hospitalaria }\end{array}$ & Europeu/Espanha & Triagem Nutricional & MAN $^{\mathrm{a}}$ & $<17$ & $54,4 \%$ \\
\hline Bartholomeyczik et al, 2010 & Gesundheitswesen & Europeu/Alemanha & Triagem Nutricional & MAN $^{\mathrm{a}}$ & $<17$ & $26,0 \%$ \\
\hline Estrada et al, 2011 & Biomedica & Americano/Colombia & Triagem Nutricional & MAN $^{a}$ & $<17$ & $54,3 \%$ \\
\hline DeLuis et al, 2011 & Nutr Hosp & Europeu/Espanha & $\begin{array}{l}\text { Triagem Nutricional } \\
\text { Bioquímicos }\end{array}$ & $\begin{array}{c}\text { MAN }^{a} \\
\text { Albumina Sérica } \\
\text { Transferrina }\end{array}$ & $\begin{array}{c}<17 \\
3,5-4,5 \mathrm{~g} / \mathrm{dl} \\
200-400 \mathrm{mg} / \mathrm{dl}\end{array}$ & $\begin{array}{c}57,9 \% \\
\text { Não apresentado } \\
\text { Nao apresentado }\end{array}$ \\
\hline Khater e Abouelezz, 2011 & $\begin{array}{c}\text { The Jornal of Nutrition, } \\
\text { Health \& Aging }\end{array}$ & Africano/Egito & Triagem Nutricional & $\mathrm{MAN}^{\mathrm{a}}$ & $<17$ & $10,8 \%$ \\
\hline Van Nie-Visser et al, 2011 & Journal of Clinical Nursing & $\begin{array}{l}\text { Europeu/Holanda e } \\
\text { Alemanha }\end{array}$ & $\begin{array}{l}\text { Antropométricos } \\
\text { Consumo }\end{array}$ & $\begin{array}{l}\text { Combinação IMC b } \\
<20 \mathrm{~kg} / \mathrm{m} 2 \text { e perda } \\
\text { de peso de } 6 \mathrm{k} \text { em } 6 \\
\text { meses e redução do } \\
\text { consumo alimentar }\end{array}$ & $\begin{array}{l}\text { Combinação IMC < } \\
20 \mathrm{~kg} / \mathrm{m} 2 \text { e perda } \\
\text { de peso de } 6 \mathrm{k} \text { em } 6 \\
\text { meses e redução do } \\
\text { consumo alimentar }\end{array}$ & $26,80 \%$ \\
\hline Santomauro et al, 2011 & $\begin{array}{c}\text { The Jornal of Nutrition, } \\
\text { Health \& Aging }\end{array}$ & Europeu/Itália & Triagem Nutricional & $\mathrm{MAN}^{\mathrm{a}}$ & $<17$ & $22,5 \%$ \\
\hline Machado e Coelho, 2011 & $\begin{array}{c}\text { The Jornal of Nutrition, } \\
\text { Health \& Aging }\end{array}$ & Americano/Brasil & $\begin{array}{l}\text { Antropométricos } \\
\text { Triagem Nutricional }\end{array}$ & $\begin{array}{l}\mathrm{IMC}^{\mathrm{b}} \\
\mathrm{PC}^{\mathrm{i}} \\
\mathrm{MAN}^{\mathrm{a}}\end{array}$ & $\begin{array}{c}<20,0 \mathrm{~kg} / \mathrm{m}^{2} \\
<31 \mathrm{~cm} \\
<17\end{array}$ & $\begin{array}{c}10,0 \% \\
34,3 \% \\
8,3 \%\end{array}$ \\
\hline Vikstedt et al, 2011 & $\begin{array}{l}\text { Journal of the American Me- } \\
\text { dical Directors Association }\end{array}$ & Europeu/Finlândia & $\begin{array}{l}\text { Triagem Nutricional } \\
\text { Consumo }\end{array}$ & $\begin{array}{c}\text { MAN }^{\mathrm{a}} \\
\text { Combinação MAN } \text { M }^{\mathrm{a}} \\
\text { redução do consumo } \\
\text { alimentar }\end{array}$ & $\begin{array}{l}<17 \\
\text { Combinação MAN e } \\
\text { reduçãp de consumo } \\
\text { alimentar }\end{array}$ & $21 \%$ \\
\hline López-Contreras et al, 2012 & $\begin{array}{c}\text { Journal of Human Nutrition } \\
\text { and Dietetics }\end{array}$ & Europeu/Espanha & $\begin{array}{l}\text { Antropomédicos } \\
\text { Consumo } \\
\text { Bioquímicos }\end{array}$ & $\begin{array}{c}\mathrm{IMC}^{\mathrm{b}}, \mathrm{PCT}^{\mathrm{f}}, \mathrm{PB}^{\mathrm{c}}, \mathrm{PMB}^{\mathrm{e}} \\
\text { Registro alimentar } \\
\text { pesado } \\
\text { Colesterol } \\
\text { Albumina Sérica } \\
\text { Contagem de Lin- } \\
\text { fócitos }\end{array}$ & $\begin{array}{l}\text { Combinação e IMC, } \\
\text { PCT, CB, CMB redução } \\
\text { do consumo alimentar } \\
\text { e } 35-53 \mathrm{~g} / \mathrm{L}^{-1} 150- \\
230 \mathrm{mg} / \mathrm{dL}\end{array}$ & $\begin{array}{l}\text { Variou de } 2 \% \text { a } \\
57 \% \text { dependendo } \\
\text { do critério diag- } \\
\text { nóstico (combi- } \\
\text { nação de todos } \\
\text { os parâmetros) }\end{array}$ \\
\hline Kshetrimayum et al, 2012 & Gerodontology & Asiático/Índia & Triagem Nutricional & MAN $^{\mathrm{a}}$ & $<17$ & 15,6 \\
\hline Rambousková et al, 2013 & $\begin{array}{c}\text { Annals of Nutrition and } \\
\text { Metabolism }\end{array}$ & $\begin{array}{c}\text { Europeu/República } \\
\text { Checa }\end{array}$ & $\begin{array}{l}\text { Antropomédicos } \\
\text { Triagem Nutricional } \\
\text { Bioquímicos }\end{array}$ & $\begin{array}{c}\text { IBC }^{\mathrm{b}} \\
\text { MAN }^{\mathrm{a}} \\
\text { Albumina } \\
\text { Pré albumina } \\
\text { Transferin } \\
\text { Urea } \\
\text { Creatina }\end{array}$ & $\begin{array}{c}<20,0 \mathrm{Kg} / \mathrm{m}^{2} \\
<17 \\
35.0-50.0 \mathrm{~g} / \mathrm{l} \\
2.00-0,40 \mathrm{~g} / \mathrm{l} \\
2.00-4.00 \mathrm{~g} / \mathrm{l} \\
2.83-8.35 \mathrm{mmol} / 171 . \\
0-1333.0 \mathrm{umol} / \mathrm{l}\end{array}$ & $\begin{array}{c}10,2 \% \\
\text { combinação de } \\
\text { todos os parâ- } \\
\text { metros }\end{array}$ \\
\hline Carlsson et al, 2013 & $\begin{array}{l}\text { Journal of Nutrition, Health } \\
\text { and Aging, }\end{array}$ & Europeu/Suécia & Triagem Nutricional & $\mathrm{MAN}^{\mathrm{a}}$ & $<17$ & $15,0 \%$ \\
\hline
\end{tabular}

Legenda: a- Mini Avaliação Nutricional (MAN); b- Índice de Massa Corporal (IMC); c- Perímetro do Braço (PB); d- Perímetro da Panturrilha (PP); e- Perímetro Muscular do Braço; f- Prega Cutânea Tricipital (PCT); g- Avaliação Subjetiva Global (ASG); h- Malnutrition Universal Screening Tool (MUST); i- Perímetro da Cintura (PC).

J. Health Biol Sci. 2014; 2(3):135-141 
ao utilizar o indicador Perímetro Muscular do Braço (PMB), adotando como ponto de corte o percentil $\leq 25$. Ressalta-se que o estudo de Gaskill et a $^{17}$, realizado na Austrália, também encontrou uma prevalência de desnutrição elevada (49,50\%), sendo o único estudo que utilizou o método de triagem Avaliação Subjetiva Global (ASG), como forma de diagnosticar a desnutrição.

Apenas o estudo de Hewitt et $\mathrm{al}^{14}$, desenvolvido na Guiana, comparou a prevalência de desnutrição entre instituições públicas $(29,3 \%)$ e particulares $(19,2 \%)$ obtidas por meio da associação entre os indicadores antropométricos como o Índice de Massa Corporal (IMC) Perímetro Braquial (PB) e Perímetro da Panturrilha (PP).

Todos os estudos utilizaram o IMC para diagnóstico do estado nutricional, seja como método isolado, ou associado a outro método, utilizando distintos valores de referência. Apenas dois estudos $22,26(9,5 \%)$ utilizaram a combinação dos dados bioquímicos com o IMC para diagnóstico da desnutrição apresentando prevalência de 10,2\% e 57,0\% (tabela 1)

No que diz respeito à utilização da MAN como método diagnóstico para a desnutrição, constatou-se que 15 estudos $(71,4 \%)$ utilizaram este questionário de triagem nutricional, e os resultados mostram que a prevalência de desnutrição variou de $6,3 \%$ a $81,7 \%$ (tabela 1 ).

\section{DISCUSSÃO}

Os dados sintetizados nessa revisão trazem algumas informações importantes: a) há grande variação na prevalência de risco de desnutrição em idosos institucionalizados; b) a maioria dos estudos sobre esta temática foi realizada a partir do ano de 2000 ; c) observaram-se diferenças nos métodos, indicadores e critérios utilizados para diagnosticar a desnutrição; d) Apenas dois estudos brasileiros abordaram a temática da desnutrição ${ }^{27,31}$.

A prevalência de desnutrição descrita pelos estudos revisados foram superiores às encontradas em ambiente hospitalar ${ }^{32}$, conforme critérios utilizados. Tal fato pode ser justificado pelas características de morbidade comum à população idosa e a dificuldade de acesso aos serviços de saúde, somadas à possível precariedade dos serviços de alimentação e nutrição das instituições asilares que, muitas vezes, não atendem às necessidades nutricionais, sensoriais e sociais desse grupo ${ }^{2}$.

É comum alguns pacientes serem admitidos em um hospital com desnutrição e outros a desenvolverem após internação ${ }^{33}$. Caso semelhante acontece com idosos que vivem em instituições asilares que, normalmente, pelas condições sociais de abandono anteriores à institucionalização, desenvolvem diversos problemas de saúde e um estado nutricional inadequado, culminando com a hospitalização.

Diversos fatores contribuem para o desenvolvimento do quadro de desnutrição em idosos institucionalizados, os quais podem estar presentes de forma isolada ou simultânea ${ }^{25}$. Os mais frequentes incluem, além da significante redução do apetite e da dificuldade de absorção de nutrientes, a excessiva restrição alimentar, diarreia crônica ou vômitos frequentes, depressão, número de dentes reduzido e próteses dentárias inadequadas, monotonia alimentar e diminuição das funções cognitivas e motoras $^{14,19,20}$.

A realização de estudos que visem conhecer o estado nutricional de idosos ao iniciar sua nova vida nessas instituições, bem como as relações que estabelecem com a família e com diversos outros aspectos sociais, podem ser determinantes para o conhecimento da causalidade e probabilidade da desnutrição vir a ser instalada.

As condições de vida dos idosos institucionalizados também precisam ser investigadas, pois a prevalência de desnutrição encontrada nos estudos revisados foi bastante elevada, principalmente no estudo de Menezes e Marucci ${ }^{27}$. Os resultados indicam, pela elevada prevalência de desnutrição, que os idosos que vivem em instituições asilares estão expostos a condições de risco nutricional.

O risco nutricional é avaliado pela combinação de estado nutricional atual e da gravidade da doença, sendo primeiro composto principalmente pelas variáveis: IMC, perda de peso recente e ingestão dietética durante a última semana de admissão ${ }^{34}$. Porém, nesses grupos é necessário que se avaliem as condições de moradia, alimentação e outros determinantes sociais, para que o diagnóstico seja mais completo e se desdobre em intervenções em saúde.

No estudo de Van Nie-Visser et $a^{23}$, apesar de apresentar a prevalência de desnutrição em seus resultados, os autores avaliaram o risco de desnutrição, uma vez que analisaram os indicadores IMC e perda de peso recente. Fato semelhante ocorreu no estudo de Lelovics et $\mathrm{al}^{12}$ desenvolvido na Hungria, que utilizou o método de triagem nutricional MUST e apresentou em seus resultados a prevalência da desnutrição.

A mesma crítica aplica-se aos estudos de Bourdel-Marchasson et al15; Smoliner et $\mathrm{al}^{19}$; Bartholomeyczik et $\mathrm{al}^{16}$; Estrada et al21; Khater e Abouelezz ${ }^{28}$; Santomauro et $\mathrm{al}^{23}$, Kshetrimayum et $\mathrm{al}^{30}$; Carlsson et $\mathrm{al}^{18}$; Centurión et al20 que utilizaram métodos isolados para o diagnóstico de desnutrição, quando deveriam apresentar resultados como a prevalência de risco de desnutrição. De acordo com a revisão realizada por Raslan et al $^{32,}$ os métodos utilizados em todos os estudos revisados se enquadram como instrumentos de triagem nutricional, e, portanto, só avaliam o risco de desnutrição.

A triagem nutricional consiste na realização de inquérito simplificado com o propósito de indicar o risco nutricional. Identifica risco de desnutrição, mudanças na condição que afetam o estado nutricional do doente, fatores que possam ter como consequências problemas relacionados à nutrição ${ }^{32}$. Ressalta-se que, nos pacientes idosos, a identificação do risco nutricional é de extrema importância para viabilizar intervenções precoces que auxiliem na manutenção do estado 
nutricional adequado, evitando que a desnutrição se instale e piore o prognóstico do paciente.

Assim, a triagem nutricional detecta a presença de risco de desnutrição, enquanto que a avaliação nutricional, além de detectar desnutrição, também classifica seu grau e permite coleta de informações que auxiliem na recuperação da saúde ${ }^{9,11}$.

Dessa forma, entendendo a complexidade das alterações que acompanham o processo de envelhecimento, são necessários critérios diagnósticos mais robustos para a avaliação do estado nutricional, com a utilização de indicadores sobre história médica, nutricional e medicamentosa, exame físico, medidas antropométricas e exames laboratoriais, resultando em um diagnóstico mais preciso ${ }^{33}$, fundamentais para o direcionamento da conduta clínica bem como para a formulação de estratégias de intervenção em saúde pública.

Em estudos epidemiológicos, a MAN vem sendo utilizada como instrumento para determinação de prevalência de desnutrição, pelo fato de que sua estrutura original, contém alguns dos critérios de triagem e avaliação, sendo também um método barato e de fácil aplicabilidade, além de identificar pessoas em estado de risco e fornecer informações necessárias para um plano de intervenção, sem a necessidade de exames laboratoriais ${ }^{16,20,22}$. No entanto, esse método não atende aos critérios para assumir o papel de uma avaliação nutricional completa.

A Avaliação Subjetiva Global (ASG) foi outro método de triagem nutricional encontrado nesta revisão ${ }^{17}$ e também foi utilizada para o diagnóstico da desnutrição. Contudo, assim como a MAN, a ASG necessita da associação de outros indicadores para um melhor diagnóstico nutricional. Ademais, esta avaliação foi desenvolvida para a triagem nutricional de pacientes hospitalizados, e não idosos institucionalizados, devendo ser aplicada em até 72 horas após a internação ${ }^{22}$. A recomendação de um período máximo de tempo para a utilização deste método pode ser um fator limitante de sua aplicação nas instituições asilares. $\mathrm{O}$ uso deste instrumento em idosos institucionalizados por um período superior a 72 horas, pode vir a comprometer a qualidade do diagnóstico e inviabilizar a triagem nutricional.

Por meio da presente revisão foi possível, também, identificar três critérios de classificação do estado nutricional antropométrico utilizando como indicador o IMC $\mathbf{I M}^{8,11,14,27,29,31}$. Entre esses, o critério mais utilizado corresponde ao ponto de corte de $20 \mathrm{~kg} / \mathrm{m}^{2}$, adotado na prática clínica geriátrica para o diagnóstico do risco nutricional35. Diante da ausência de um consenso sobre o melhor ponto de corte adotado para a classificação do IMC, destaca-se, mais uma vez, a importância da associação com outros indicadores do estado nutricional para a avaliação do risco e do diagnóstico da desnutrição.

\section{CONSIDERAÇÕES FINAIS}

A partir desta revisão sistemática, constatou-se uma elevada prevalência de desnutrição $(81,7 \%)$ em idosos institucionalizados, sendo a MAN o método mais utilizado para seu diagnóstico. No entanto, mais estudos são necessários para o desenvolvimento de um instrumento que permita uma avaliação nutricional mais completa, com critérios uniformes, de baixo custo e fácil aplicabilidade, que possa ser utilizado tanto para o acompanhamento clínico quanto para a investigação epidemiológica.

Este estudo de revisão pode auxiliar os profissionais que atuam na área da gerontologia e geriatria a reorientarem as suas práticas em saúde contribuindo para uma melhor qualidade de vida desta população.

\section{AGRADECIMENTOS}

À Fundação de Apoio à Pesquisa do Estado do Rio Grande do Norte (FAPERN) e à Coordenação de Aperfeiçoamento de Pessoal de Nível Superior (CAPES) pelo financiamento e bolsas aos pesquisadores.

\section{REFERÊNCIAS}

1. Giacomin, KC. Envelhecimento populacional e os desafios para as políticas públicas. In: Bernizis MV, Borges, MC. Políticas Públicas para um país que envelhece. São Paulo: Martinari; 2012. 31p.

2. Santana IO, Coutinho MPL, Ramos N, Santos DS, Lemos GLC, Silva PB. Mulher Idosa: Vivências do Processo de Institucionalização. Ex aequo [Internet]. 2012 [citado em: 2014 Fev 12]; (26): 71-85. Disponível em http://www.scielo.gpeari. mctes.pt/scielo.php?script $=$ sci_arttext\&pid=S0874-55602012000200007\&lng $=p$ t\&nrm=iso

3. Brasil. Agência Nacional de Vigilância Sanitária. Resolução nº 283, de 26 de setembro de 2005. Regulamento Técnico que define as normas de funcionamento para as Instituições de Longa Permanência para Idosos. Diário Oficial [da] República Federativa do Brasil. 2005 set. 27; Seção 1. p. 58-59.

4. Santelle O, Lefèvre AMC, Cervato AM. Alimentação institucionalizada e suas representações sociais entre moradores de instituições de longa permanência para idosos em São Paulo, Brasil. Cad. Saúde Pública [Internet]. 2007 Dez [citado em: 2013 Março 15]; 23(12): 3061-5. Disponível em: http://www.scielo.br/pdf/ csp/v23n12/28.pdf.

5. Campos MTS, Monteiro JBRE, Ornelas APRC. Fatores que afetam o consumo alimentar e a nutrição no idoso. Rev Nutr. 2000; 13(3):157-65. doi: http://dx.doi. org/10.1590/S1415-52732000000300002.

6. Sullivan, D. H; SUN, S; Walls, R. C. Protein-energy undernutrition among elderly hospitalized patients. JAMA. 1999 Jun 2; 281(21): 2013-9. PMID: 10359390.

7. Del Álamo, C, Rodicio SG, Freile BC, Pérez LA, Pérez J, Pindado MAC. Protocolo de valoración, seguimiento y actuación nutricional em um centro residencial para personas mayores. Nutr Hosp. [Internet] $2008 \mathrm{Mar}-\mathrm{Abr}$ [citado em: 2013, Março, 20]; 23(2): 100-04. Disponível em: http://scielo.isciii.es/scielo. php?script=sci_arttext\&pid=S0212-16112008000200005

8. Van Nie-Visser NC, Meijers JM, Schols JM, Lohrmann C, Bartholomeyczik S, Halfens RJ. Comparing quality of nutritional care in Dutch and German nursing homes. J Clin Nurs. 2011 Sep; 20(17-18): 2501-08. doi: 10.1111/j.1365- 
2702.2011.03761.x. PMID: 21722223.

9. Wojszel ZB. Determinants of nutritional status of older people in long-term care settings on the example of the nursing home in Białystok. Adv Med Sci. 2006; 51:168-173. PMID: 1735730.

10. Carlsson. M, Haglin L, Rosendahl E, Gustafson Y. Poor nutritional status is associated with urinary tract infection among older people living in residential care facilities. J Nutr Health Aging. 2013 Feb; 17(2): 186-91. PMID: 23364500.

11. Langiano E, Di Russo C, Atrei P, Ferrara M, Allegretti V, Verdicchio I, De Vito E. Nutritional status of elderly institutionalized subjects in a health district in Frosinone (Italy). Ig Sanita Pubbl. 2009 Jan-Feb; 65(1):17-28. PMID: 19494920.

12. Von Elm E, Altman DG, Egger M, Pocock SJ, Gotzsche PC, Vandenbroucke JP. Strengthening the Reporting of Observational Studies in Epidemiology (STROBE) statement: guidelines for reporting observational studies. BMJ.2007 Oct 20; 335(7624):806-8. PMID: 17947786.

13. Sampaio, R.F e Mancine, M.C. Estudos de revisão sistemática: um guia para síntese criteriosa da evidência científica. Rev. bras. Fisioter [Internet] 2007 JanFev [citado em: 2013, Março, 15]; 11(1): 83-89. Disponível em: http://www.scielo. $\mathrm{br} / \mathrm{pdf} / \mathrm{rbfis} / \mathrm{v} 11 \mathrm{n} 1 / 12$.pdf.

14. Hewitt, G, Smail S, Patterson S, Draper A. The Nutritional Vulnerability of Older Guyanese in Residential Homes. West Indian Med J. 2006 Oct; 55(5): 334. doi: http://dx.doi.org/10.1590/S0043-31442006000500009

15. Bourdel-Marchasson, Rolland C, Jutand MA, Egea C, Baratchart B, Barberger-Gateau P. Undernutrition in geriatric institutions in South-West France:Policies and risk factors. Nutrition. 2009 Feb; 25(2):155-164. doi: 10.1016/j.nut.2008.07.016. PMID: 18848433.

16. Bartholomeyczik S, Reuther S, Luft L, van Nie N, Meijers J, Schols J, et al. Prevalence of malnutrition, interventions and quality indicators in German nursing homes - First results of a nationwide pilot study. Gesundheitswesen. 2010 Dec; 72 (12): 868-874. doi: 10.1055/s-0029-1246150. PMID: 2221991.

17. Gaskill D, Black LJ, Isenring EA, Hassall S, SandersF, Bauer JD. Malnutrition prevalence and nutrition issues in residential aged care facilities. Australa J Ageing. 2008 Dec; 27(4): 189-194. doi: 10.1111/j.1741-6612.2008.00324.x. PMID: 1032620.

18. Carlsson M, Gustafson Y, Eriksson S, Haglin L. Body composition in Swedish old people aged 65-99 years, living in residential care facilities. Arch Gerontol Geriatr. 2009 Ju-Aug; 49(1): 98-107. doi: 10.1016/j.archger.2008.05.012. PMID: 1870340 .

19. Smoliner C, Norman K, Wagner KH, Hartig W, Lochs H, Pirlch M. Malnutrition and depression in the institutionalised elderly. Br J Nutr. 2009 Dec; 102(11):1663-1667. doi: 10.1017/S0007114509990900. PMID: 19622192.

20. Centurión L, Vizuete AA, Anta RMO, Rodríguez ER, Plaza BL, Sobaler AML. Asociacion entre riesgo de desnutricion y capacidad afectiva en personas de edad avanzada institucionalizadas de la Comunidad de Madrid. Nutr Clin Diet Hosp. [Internet] 2010 [cited 2013, Março, 20,]; 30(2):33-4. Availabe: http://dialnet. unirioja.es/servlet/articulo? codigo $=3311292$.

21. Estrada A, Cardona D, Segura AM, Chavarriaga LM, Ordóñez J, Osorio JJ. Calidad de vida de los adultos mayores de Medellín. Biomédica. 2011. 31(4): 492502. doi: http://dxdoi.org/10.7705/biomedica.v31i4.399.

22. De Luis DA, Lopez Mongil R, Gonzalez Sagrado M, Lopes Trigo JA, Mora
PF, Castrodeza Sanz J. Evaluation of the mini-nutritional assessment short-form (MNA-SF) among institutionalized older patients in Spain. Nutr Hosp. 2011 Nov-Dec; 26(6):1350-1354.PMID: 22411382.

23. Santomauro F, Olimp N,Baggiani L, Comodo N, Mantero S, Bonaccorsi G. Bioelectrical impedance vector analysis and mini nutritional assessment in elderly nursing home residents. J Nutr Health Agng. 2011 Mar; 15(3): 163-167. PMID: 21369661.

24. Vikstedt T, Suominen MH, Joki A, Muurinen S, Soini H, Pitkealea KH. Nutritional Status, Energy, Protein, and Micronutrient Intake of Older Service House Residents. J Am Med Dir Assoc. 2011 May; 12(4): 302-307. doi: 10.1016/j. jamda.2010.12.098. PMID: 217172.

25. Lopez-Contrera M.J, Torralba C, Zamora S, Pérez-Llamas F. Nutrition and prevalence of undernutrition assessed by different diagnostic criteria in nursing homes for elderly people. J Hum Nutr Diet. 2012 Jun;25(3): 239-246. doi: 10.1111/j.1365-277X.2012.01237.x. PMID: 22360373.

26. Rambousková J, Slaviková M, Krsková A, Procházka B, Andel M, Dlouhý P. Nutritional Status Assessment of Institutionalized Elderly in Prague, Czech Republic. Ann Nutr Maetab. 2013;62(3):199-204. PMID: 23485785.

27. Menezes, T. N., Marucci, M.F.N. Avaliação antropométrica de idosos residentes em instituições de longa permanência de Fortaleza-Ce. Rev Bras Geriatr Gerontol. 2010; 13(2):235-243.

28. Khater MS, Abouelezz NF. Nutritional status in older adults with mild cognitive impairment living in elderly homes in Cairo, Egypt. J Nutr Health Aging. 2011 Feb; 15(2):1350-1354. PMID: 21365162.

29. Lelovics Z, Bozó RK, Lampek K, Figler M. Results of nutricionalscreenig in institutionalized elderly in Hungary. Arch Gerontol Geriatr. 2009 Jul-Ago; 49(1):190-6. doi: 10.1016/j.archger.2008.07.009. Epub 2008 Sep 10. PMID: 18786738 .

30. Kshetrimayum N, Reddy CV, Siddhana S, Manjunath M, Rudraswany S, Sulavai S. Oral health-related quality of life and nutritional status of institutionalized elderly population aged 60 years and above in Mysore City, India. Gerodontology. 2012 Feb 26; 30(2):119-25. PMID: 22364560.

31. Machado RSP. Coelho MASC. Risk of malnutrition among Brazilian institutionalized elderly: a study with the Mini Nutritional Assessment (MNA) questionnaire. J Nutr Health Agin. 2011 Aug; 15(7):532-5. PMID: 21808930.

32. Raslan M, Gonzalez MC, Dias MCG, Barbosa CP Cecconello I, Waitzberg DL. Aplicabilidade dos métodos de triagem nutricional no paciente hospitalizado. Ver Nutri. 2008 Sep-Out; 21(5):553-61. doi:http://dx.doi.org10.1590/S1415523200800050008 .

33. Sungurtekin H, Sungurtekin U, Balci C, Zencir M, Erdem E. The influence of nutritional status on complications after major intraabdominal sugery. J. Am. Coll Nutr. 2004 Jun 23(3):227-32. PMID: 15190047.

34. Johanse .N, Kandrup J, lum LM, Bak L, Norregaard P, Bunch E, et al. Effect of nutritional support on clinical outcome in patients at nutritional risk. Clin Nutri. 2004 Aug; 23(4):539-50. PMID: 1529709.

35. Perissinotto E, Pisent C, Sergi G, Grigoletto F; ILSA Working Group. Anthropometric measurements in the elderly: age and gender differences. Br J Nutr 2002 Feb; 87(2):177-86. PMID: 11895170. 